\title{
Impact of postglacial warming on borehole reconstructions of last millennium temperatures
}

\author{
V. Rath ${ }^{1}$, J. F. González Rouco ${ }^{1}$, and H. Goosse ${ }^{2}$ \\ ${ }^{1}$ Universidad Complutense de Madrid, Facultad CC. Físicas, Departamento de Astrofísica y CC. de la Atmósfera, \\ Ciudad Universitaria, 28040 Madrid, Spain \\ ${ }^{2}$ Centre de recherches sur la terre et le climat Georges Lemaître, Earth and Life Institute, Université Catholique de Louvain, \\ 2 chemin du Cyclotron, 1348 Louvain-la-Neuve, Belgium
}

Correspondence to: V. Rath (vrath@ fis.ucm.es)

Received: 8 September 2011 - Published in Clim. Past Discuss.: 12 October 2011

Revised: 21 May 2012 - Accepted: 31 May 2012 - Published: 19 June 2012

\begin{abstract}
The investigation of observed borehole temperatures has proved to be a valuable tool for the reconstruction of ground surface temperature histories. However, there are still many open questions concerning the significance and accuracy of the reconstructions from these data. In particular, the temperature signal of the warming after the Last Glacial Maximum is still present in borehole temperature profiles. It is shown here that this signal also influences the relatively shallow boreholes used in current paleoclimate inversions to estimate temperature changes in the last centuries by producing errors in the determination of the steady state geothermal gradient. However, the impact on estimates of past temperature changes is weaker. For deeper boreholes, the curvature of the long-term signal is significant. A correction based on simple assumptions about glacial-interglacial temperature changes shows promising results, improving the extraction of millennial scale signals. The same procedure may help when comparing observed borehole temperature profiles with the results from numerical climate models.
\end{abstract}

\section{Introduction}

In steady state conditions, with constant thermal conductivity and neglecting heat production, the subsurface temperature depth profile is linear (e.g. Pollack and Huang, 2000). If perturbations in surface temperatures occur, they propagate to the subsurface and deform the geothermal gradient. Such deviations are recorded by borehole temperature profiles (BTPs) and have been successfully used to reconstruct ground surface temperature histories (GSTHs), providing estimates of preindustrial-to-present temperature change that complement those of other proxy reconstructions (e.g. Jansen et al., 2007). Nevertheless, there are still a number of open questions concerning possible biases in borehole based reconstructions (e.g. Smerdon et al., 2006; Wilhelm et al., 2005; Verdoya et al., 2007; Mottaghy and Rath, 2006), making the choice of boreholes, the careful evaluation of their setup, and a valid treatment of the data a considerable task for the interpreter.

One such source of uncertainty concerns the potential effect of long term surface perturbations like the warming from the Last Glacial Maximum (LGM) to the Holocene. Though reliable conclusions about the last $25000 \mathrm{yr}$ can only be drawn using subsurface temperatures from the rare deep boreholes with depths of $2000 \mathrm{~m}$ and more (e.g. Chouinard and Mareschal, 2009), at depths shallower than $1000 \mathrm{~m}$, the post-LGM warming may also leave an imprint through smooth changes of the temperature gradient. Within this depth domain, there is an abundance of BTPs which could be a valuable source of information on the last millennium and late Holocene. The extent to which such perturbations can affect the interpretation of past temperature inversion from BTPs within this range of depths is unclear.

Since the time of Birch (1948), corrections for paleoclimate effects have been proposed and applied when estimating heat flow densities (e.g. Vasseur and Lucazeau, 1983; Majorowicz and Wybraniec, 2010), though this is not yet the standard procedure (Davies and Davies, 2010). Surprisingly, the role of postglacial warming in the reconstruction of past 
climates has not yet been studied systematically, though its effect was identified as a source of error when inverting very shallow borehole temperature profiles many times (amongst others Majorowicz, 2004; Hartmann and Rath, 2005; Beltrami et al., 2011). In this paper we show that the analysis of its impact is even more important when interpreting deeper boreholes. Important implications may be expected not only for derived temperatures, stored heat, and heat flow estimations, but also when comparing observed BTPs and model simulations (see González-Rouco et al., 2009, and references therein). Therefore, it would be highly desirable to estimate the influence of postglacial warming on BTPs of variable depths within the first kilometre of the subsurface and, if possible, formulate approaches for dealing with this situation.

This work analyzes these issues by performing MonteCarlo (MC) simulations with a one-dimensional forward modeling code under plausible glacial-interglacial surface temperature forcing conditions described in Sect. 2, demonstrating the effects of postglacial warming on shallow BTPs. In Sect. 3 we develop simple corrections that mitigate these effects. Appendices A and B give further information on the numerical procedure which is used to produce the Monte Carlo (MC) results, and the inverse procedure employed. The MATLAB ${ }^{\mathrm{TM}}$ scripts used for the MC simulations and numerical inverse experiments may be downloaded as a supplement to this article.

\section{Monte-Carlo simulations}

In order to give a quantitative estimate of the effect of the postglacial temperature rise, $\mathrm{MC}$ simulations were performed. For this we employed a simple numerical onedimensional forward modeling code (Mottaghy and Rath, 2006; Rath and Mottaghy, 2007), which is described in more detail in Appendix A. To represent the past temperature variations, a simplified upper temperature boundary condition was used. It is based on a sequence of step functions as shown in Fig. 1. The parameters sampled in the MC calculations are the times of temperature changes $t_{1}$ and $t_{2}$, and the corresponding temperature deviations $\Delta T_{1}$ and $\Delta T_{2}$, respectively. $\Delta T_{1}$ may be described as the temperature rise between glacial and Holocene conditions, while $\Delta T_{2}$ represents the difference between Holocene and the long-term Quaternary mean. We assumed parameter distributions for location and amplitude of both step functions, as well as for the petrophysical properties, the thermal conductivity and the volumetric heat capacity, $\lambda_{m}$ and $(\rho c)_{m}$ of the rock, respectively.

The resulting six parameters were assumed to be independently distributed following a Normal distribution $\mathcal{N}(\hat{\mu}, \hat{\sigma})$. The values assumed for the means $\hat{\mu}$ and standard deviations $\hat{\sigma}$ are given in Appendix A. This set of parameters was randomly sampled, producing an ensemble of 10000 runs of the forward modeling code.

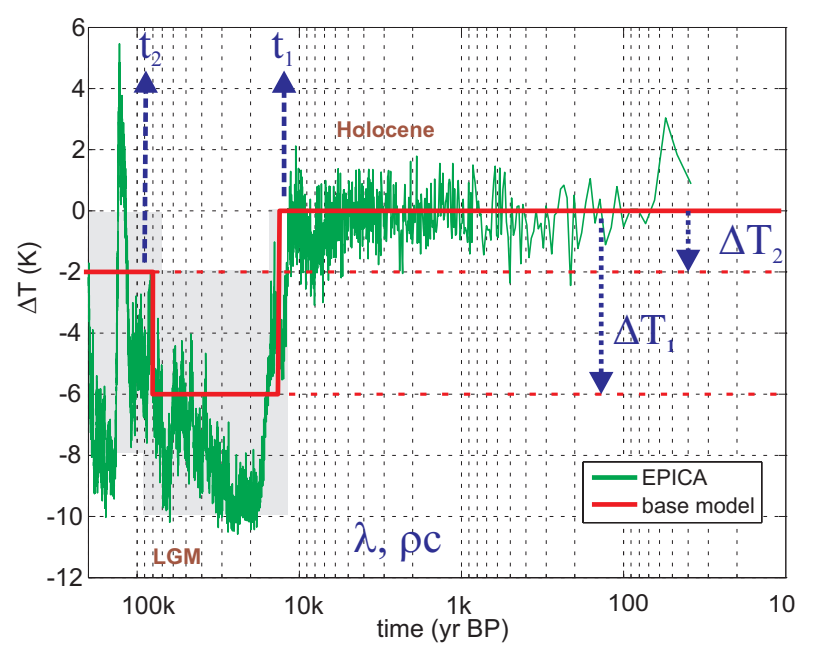

Fig. 1. GSTH forcing used for the calculations presented in this study. Also shown are the parameters and their variations used for the Monte Carlo investigations. $2 \hat{\sigma}$ boundaries are marked by grey shades. The general shape of the GSTH for this numerical experiment is motivated by the EPICA ice core reconstructions (Jouzel et al., 2007). Note that the base model is constant since the postglacial temperature rise.

The general shape of the step functions shown in Fig. 1 was qualitatively motivated by estimations like those of GRIP, EPICA and Vostok (e.g. NGRIP Working Group, 2004; Jouzel et al., 2007). The values are within the uncertainties of present knowledge concerning glacial to interglacial land temperature change, and embrace scenarios of less cooling like those plausibly suffered at lower latitudes as well as larger temperature changes registered in proxy data and simulated by models for northern latitudes (Jansen et al., 2007; Otto-Bliesner et al., 2009, and references therein). Some large temperature changes over land (e.g. Jost et al., 2005) were assigned low probabilities. The choice of GSTH also would not include sites from the very high latitudes, where the conditions at the base of the ice sheets may cause LGM ground surface temperatures significantly higher than during the Holocene. We think that the general shape of the GSTHs employed in this study is a reasonable choice. Note that the the proposed set of surface temperature histories (see Fig. 1) does not intend to be exhaustive in including all potential past climate trajectories, but to provide a plausible framework of past temperature change over a range of borehole locations that can be used to demonstrate its effect on BTPs. The sensitivity of downhole observations to past surface temperature variability decreases strongly going back in time. Therefore, short-period changes related to the glacial-interglacial cycle may safely be approximated by appropriate simple functions or average values as chosen here. For the far past beyond the last glacial cycle, this has been shown by Majorowicz et al. (2008). Even a variable duration of the glacial-interglacial transition does not have a 


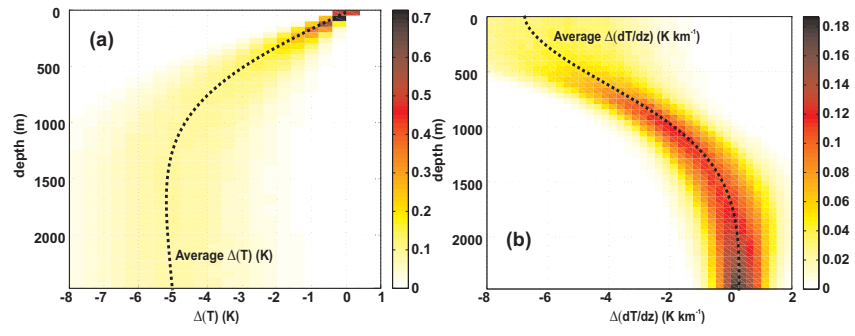

Fig. 2. Results of the Monte Carlo study. Normalized histogram densities of the temperature deviation calculated for the long-term models with respect to steady-state conditions (a), and the vertical derivative (b) of temperature as functions of depth. For all figures, the integral along the $\mathrm{x}$-axis is equal to 1 for all depths, as the densities are normalized by the number of runs $(10000)$.

meaningful effect given observational uncertainty. However, if reliable information is available on the regional climate conditions during the last glacial cycle, it could be used to further constrain the probable signature of GST changes.

Figure 2 shows the difference of the resulting BTPs with respect to the reference, i.e. a constant surface temperature of $T_{\mathrm{S}}=6^{\circ} \mathrm{C}$, implying steady-state conditions. The results indicate that the temperature profiles differ considerably from the reference by the influence of the earlier temperature changes. The largest deviations in temperature occur at depths between $1000 \mathrm{~m}$ and $1500 \mathrm{~m}$. Clearly, the true steady state condition can not easily be estimated from the temperature and thermal properties alone for a given shallow (say, $<500 \mathrm{~m}$ ) borehole, as it is often done in practice by assuming that the quasi-linear bottom part of the profile represents the steady state geothermal gradient. This may be concluded from the observation that the vertical temperature gradient approaches its steady state value only at depths near $2000 \mathrm{~m}$. It follows, that under most probable conditions, results of GSTH inversions could be influenced by miscalculating the steady-state component. Additionally, this implies that differences in log depth may falsely translate into different geothermal gradients, and in consequence, different GST histories.

\section{A simple correction approach}

Boreholes of less than $1000 \mathrm{~m}$ depth allow for targeting changes of the last several $1000 \mathrm{yr}$. It is highly desirable, however, to make better use of the information content of these shallow BTPs, as they are abundant in many areas, while deep boreholes are very rare. Therefore, a simple procedure is proposed, by which shorter BTPs may be corrected for the influence of the glacial-interglacial transition with just an approximate knowledge of the regional long-term paleo-temperatures.

A GSTH based on the major features of the Holocene warming scenario with additional temperature variations during the last $500 \mathrm{yr}$ (grey line in Fig. 3) was used to
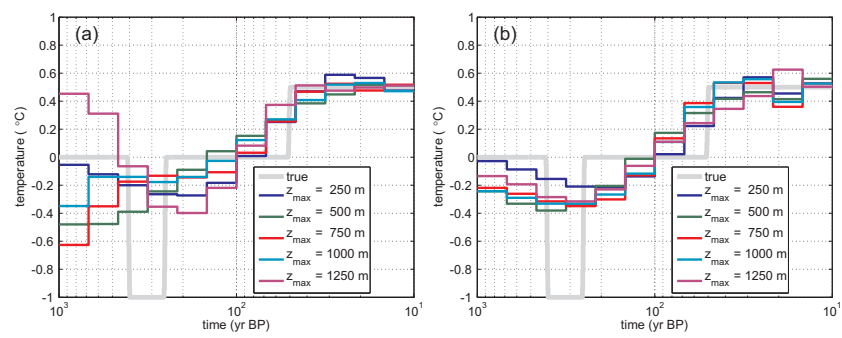

Fig. 3. Using prior knowledge for correction of shallow borehole temperature profiles. (a) Inversion of BTP of different lengths derived from a synthetic GSTH (shown in grey), which shows constant temperatures since the postglacial temperature rise. (b) Boreholes were corrected by subtracting the response to this prior GSTH. The shallowest BTP ( $250 \mathrm{~m}$, dark blue) is too short to resolve the LIAlike structure, and thus the results show only very weak effects, independent of whether input data are raw or corrected. A regularization parameter of 0.25 was used in this numerical experiment.

generate synthetic BTPs of different depth between $250 \mathrm{~m}$ and $1250 \mathrm{~m}$. This scenario includes a Little Ice Age (LIA) like minimum around ca. 1700 and a warming of about half a degree in the last century. After being artificially perturbed with noise, these synthetic observations were interpreted using a regularized linear inversion scheme (see Beltrami and Mareschal, 1991; Mareschal and Beltrami, 1992). Results show a large variability among the inverted BTPs (Fig. 3), particularly before the LIA minimum where deviations to the reference GSTH can reach about half a degree at the beginning of the millennium. This discrepancy mainly arises because of the post-glacial warming that fakes true temperature changes in the last millennium. This can then be corrected by subtracting the response corresponding to the long term component of the GSTH, which is the original GSTH with a constant temperature assumed from $12000 \mathrm{yr}$ BP onward (see Fig. 1, red line). This leads to much more consistent results for all inverted profiles for all of the millennium (Fig. 3).

Concerning the use of shallow (say, $<500 \mathrm{~m}$ ) boreholes, it has to be noted that single shallow boreholes usually still lead to meaningful short term reconstructions that reproduce the changes in the last centuries, because the disturbing temperature signal is nearly linear, and can thus easily be represented by an erroneous steady state component (i.e. the geothermal gradient) of the model. This can easily be seen in Fig. 4, where background heat flow estimated from BTPs of increasing depths is shown for a realistic profile including the post LGM warming (red), and its corrected version (green) as well as for a case including only changes in the last millennium (green; excluding post LGM warming), and for the steady state (gray). The background geothermal gradients derived from the raw profile (i.e. including LGM effects) at shallow depths are systematically too low, while the ones derived from the corrected values are very similar to the true ones. They also agree with the results for synthetic BTPs 


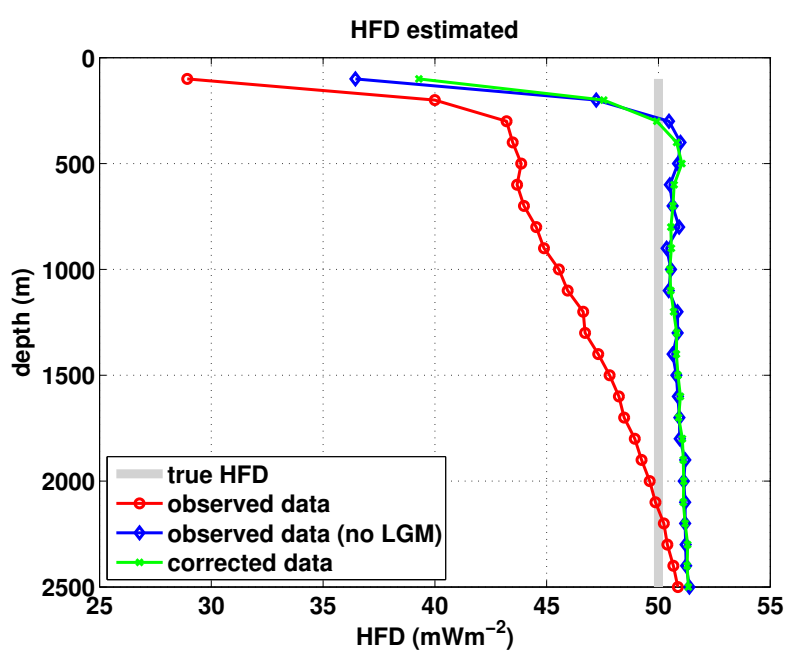

Fig. 4. Background heat flow density values obtained as a result from inverting original synthetic (red), synthetic data without LGM (blue), and corrected data (green). Obviously, in the first case the estimated heat flow density is the superposition of the nearly linear equilibrium component, and the effect of post-glacial warming. Shown in grey is the true constant value. The remaining deviation from the true one is present in the blue and green curve. It depends on the observations, their errors, and the choice of the regularization parameter (in this case $\epsilon=0.25$ ).

estimated from a reference GSTH, which is constant in time with exception of the millennial period.

For these reasons, increasing the depth into the domain of changing vertical gradient will not generally improve results. In this case, the effect of the Holocene warming may not be treated as a simple offset in basal heat flow density, but will produce an erroneous signal in the whole profile, and therefore in the reconstructed surface temperatures. If BTPs of different depths are compared, or even interpreted jointly, inconsistencies and corresponding errors in the results will arise.

Note that for the case involving an abrupt change in temperature, all inverted GSTHs, whether corrected or not, underestimate the temperature change in the last $1000 \mathrm{yr}$. This is due to the existence of many GSTHs leading to a similar fit of the observations. The inverse problem is ill-posed (Hansen, 1998, 2010), and can only be solved by regularization. The particular method used here is based on a damped singular value decomposition. As with many other commonly employed methods, it will lead to smooth solutions which will not reproduce abrupt changes like the one we have chosen for the numerical experiment. If the corresponding regularization parameter is chosen properly (see Appendix B), the effect of postglacial warming would not lead to a general overestimation of variation. Such overestimation would have explained the comparatively cold temperatures of the borehole reconstruction results presented by Jansen et al. (2007); the
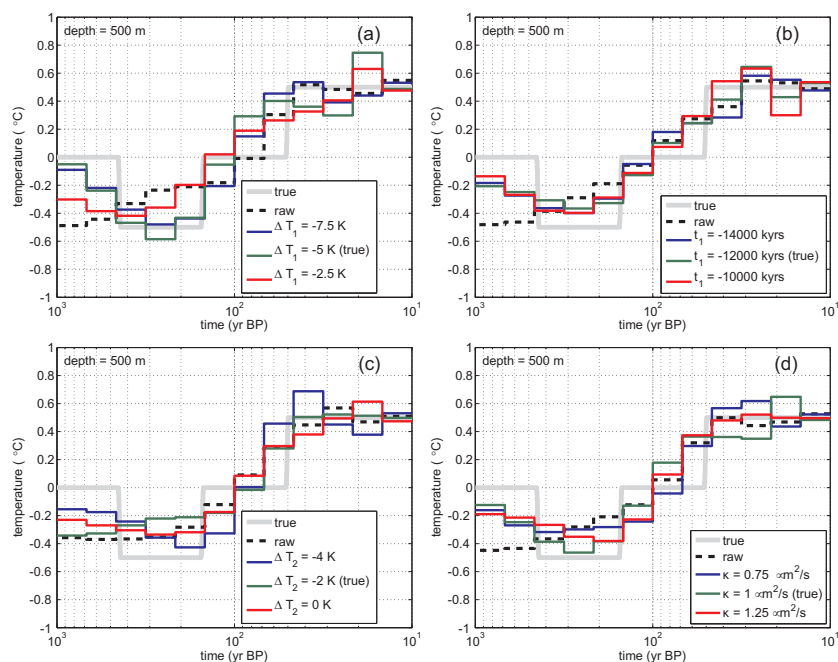

Fig. 5. Sensitivity of inversion results to different parameters used for the calculation of BTPs used for correction. The inverted BTP had a length of $500 \mathrm{~m}$, and were perturbed with normally distributed noise characterized by zero mean and a standard deviation $\hat{\sigma}=0.05 \mathrm{~K}$. Data were corrected with different paleotemperature scenarios. (a) the amplitude of LGM, $T_{1}$; (b) the corresponding step time $t_{1} ;$ (c) the preglacial long term mean $T_{2} ;$ (d) the rock thermal diffusivity $\kappa=\frac{\lambda}{\rho c}$. A regularization parameter of 0.12 was used for all inversions.

present numerical experiments, however, indicate that this hypothesis is not valid.

Clearly, the method as applied here makes use of our prior knowledge of long term GSTH, and to a lesser degree on rock properties and basal heat flow as far as nonlinearity has to be considered. However, it turns out that the results can also be improved by using approximate information. Sensitivity tests indicate that even inaccurate long-term models improve inversion results considerably. Figure 5 shows results obtained by varying the prior GSTH calculating the correction applied to the raw data. The different paleotemperature scenarios were generated varying (a) the amplitude of the LGM, $\Delta T_{1}$, with a variation of $\pm 2.5 \mathrm{~K}$; (b) the corresponding step time $t_{1}$, with a $\Delta t$ of $\pm 2 \mathrm{kyr}$; (c) the pre-glacial long term mean $\Delta T_{2}$; and (d) rock thermal diffusivity $\kappa=\frac{\lambda}{\rho c}$. The differences between the inferred GST histories are much smaller than in the case of uncorrected observations. These result indicate that even incomplete prior knowledge on paleotemperatures may improve consistency and realism of the inversion results.

Finally, it should be noted that this simple reduction approach is not the only possible one when reconstructing past surface temperature changes. In the case of Bayesian-type reconstruction algorithms (see e.g. Tarantola, 2005), the earlier surface temperature changes could be introduced as a prior model in the case of a Gaussian maximum a posteriori estimate, or included into the prior probability distribution in the more general case. 


\section{Conclusions}

From the simple modeling studies presented above a few conclusions may be drawn. First of all, the signature of the LGM and Holocene warming can not be neglected even in shallow boreholes. In this case, however, the nearly linear behavior of the LGM-derived signal component can be represented by an erroneous background equilibrium heat flow. In the case of deeper BTPs, carrying information from times before the LIA, the curvature of the perturbing signal becomes important, and differences in depth translate to variations in inferred paleotemperatures. A first order correction, however, seems possible by means of approximate knowledge on prior development of surface temperatures.

A correction of this kind could also be useful when comparing the output of numerical climate models to BTPs observed in the field. From this study it is clear that observed temperatures will commonly contain a long-term signal. To date this component is not represented in most numerical simulation codes due to their very limited treatment of subsurface heat balance processes, and particularly the assumption of a no-flux bottom boundary condition at shallow depths (Smerdon and Stieglitz, 2006; Stieglitz and Smerdon, 2007; MacDougall et al., 2010). To represent the subsurface thermal effects on the scale of $10000 \mathrm{yr}$ or more, the zero or fixed flux boundary condition should be imposed at a depth of more than $1000 \mathrm{~m}$. The model-observation comparisons published to date generate synthetic observations with offline subsurface models (see Stevens et al., 2008; González-Rouco et al., 2009) not suffering this limitations, at the price of neglecting any land-atmosphere feedback from the subsurface due to its changing heat content. The approach presented here may be an easy remedy allowing to use existing simulation tools with these data in a manner consistent with the simulated physics.

Finally, a little caveat is indicated. Let us assume that a given BTP meets the commonly accepted conditions for paleoclimatic interpretation, e.g. absence or sufficient information on advective heat transport, topography, and subsurface properties. The proposed correction requires an additional assumption, which surely implies the possibility of error. Even if the large-scale SAT development is reasonably well known, the local GSTH may differ considerably. It has to be kept in mind that subsurface temperatures record the conditions at the Earth's surface, which is not always exposed directly to atmospheric conditions. The past existence of ice sheets and their complex dynamics, flooding related to isostatic effects, glacial lakes, or even changes in vegetation may have influenced or even dominated the site GSTH since the Last Glacial Maximum. Care has to be taken to guarantee that these particular conditions are taken into account. The power of this approach has thus to be investigated in the field, which remains a task for the future.
Table A1. Parameters used for the Monte-Carlo simulations in this study. They were assumed to be independently and normally distributed. $\lambda_{m}$ and $(\rho c)_{m}$ are the rock matrix properties.

\begin{tabular}{lllllll}
\hline & $\begin{array}{l}\lambda_{m} \\
\left(\frac{\mathrm{W}}{\mathrm{mK}}\right)\end{array}$ & $\begin{array}{l}(\rho c)_{m} \\
\left(\frac{\mathrm{MJ}}{\mathrm{kgK}}\right)\end{array}$ & $\begin{array}{l}\Delta T_{1} \\
(\mathrm{~K})\end{array}$ & $\begin{array}{l}t_{1} \\
(\mathrm{kyr})\end{array}$ & $\begin{array}{l}\Delta T_{2} \\
(\mathrm{~K})\end{array}$ & $\begin{array}{l}t_{2} \\
(\mathrm{kyr})\end{array}$ \\
\hline$\hat{\mu}$ & 2.5 & 2.0 & 5.5 & 14 & 4 & 80 \\
$\hat{\sigma}$ & 0.5 & 0.5 & 2 & 2 & 2 & 10 \\
\hline
\end{tabular}

\section{Appendix A}

\section{Supplementary information on the MC simulation}

The one-dimensional, purely conductive heat equation in a porous medium can be written as

$$
\frac{\partial}{\partial z}\left(\lambda_{\mathrm{e}} \frac{\partial T}{\partial z}\right)+h=(\rho c)_{\mathrm{e}} \frac{\partial T}{\partial t}
$$

where $\lambda$ is thermal conductivity $\left(\mathrm{W} \mathrm{m}^{-1} \mathrm{~K}^{-1}\right),(\rho c)$ is the volumetric heat capacity $\left(\mathrm{J} \mathrm{K}^{-1} \mathrm{~m}^{-3}\right)$, and $h$ is volumetric heat production $\left(\mathrm{W} \mathrm{m}^{-3}\right)$. The subscript " $\mathrm{e}$ " marks effective parameters of the porous medium, and can be interpreted as properties of a two-phase mixture between solid rock and fluid-filled pore space. For the paleoclimate application we have in mind, Eq. (A1) usually is solved with appropriate boundary conditions, namely fixed but time-dependent temperature $T=T(t)$ at the top, $z=z_{0}$, and fixed heat flow density $q_{\mathrm{b}}$ at the base at $z=z_{\mathrm{b}}$.

Equation (A1) is understood to allow all coefficients, boundaries, and sources, to be nonlinearly dependent on temperature. As we are aiming at deep boreholes, recording the history of ground surface temperature for some $10000 \mathrm{yr}$, we have to extend the numerical model to depths of several $\mathrm{km}$ for numerical reasons, and temperatures of up to $200^{\circ} \mathrm{C}$ accordingly. This requires taking the temperature dependencies of the thermophysical properties into account, possibly including phase change by freezing and thawing of pore water. Details of theory, implementation, and the validation of the approach can be found in Mottaghy and Rath (2006) and Rath and Mottaghy (2007). In contrast, if aiming at millennial scale events as the Little Ice Age in Europe, analytical models (e.g. Beltrami and Mareschal, 1991; Mareschal and Beltrami, 1992, used in the inverse experiments described below) assuming constant properties are often sufficient for the interpretation of BTPs, implying additivity of solutions.

The parameter choices for the MC simulations are given in Table A1. While the rock parameters $\lambda_{m}$ and $(\rho c)_{m}$ are plausible for the most common crustal rocks. To complete the model set up, we have assumed a moderate porosity of $\phi=0.1$, a recent surface temperature of $T_{\mathrm{S}}=6^{\circ} \mathrm{C}$, and a heat flow density of $50 \mathrm{~m} \mathrm{~W} \mathrm{~m}^{-2}$. 


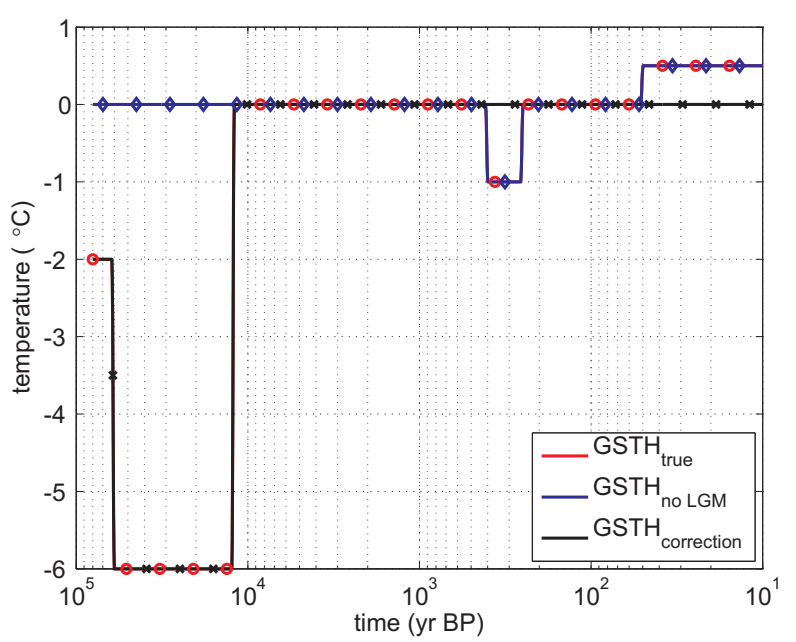

Fig. B1. GSTH used in the synthetic inversion experiments. BTPs derived from the true model (red) are named observed data, while the temperatures used for correction are derived from the black GSTH. For comparison, also synthetic observations assuming a constant behavior before the LIA are derived from the blue GSTH.

\section{Appendix B}

\section{Additional information on the inverse numerical experiments}

For the numerical experiments on GSTH inversion, we used a commonly employed procedure (Beltrami and Mareschal, 1991; Mareschal and Beltrami, 1992; Beltrami et al., 1995; Clauser and Mareschal, 1995), where a simple analytical forward solution is used, and the subsurface is assumed to be homogeneous:

$T(z, t)=T_{0}+\frac{q_{0} z}{\lambda}-\frac{A z^{2}}{2 \lambda}+T_{t}(z, t)$.

The first three terms represent the steady-state component, defined by the heat flow density at the surface $q_{0}$, the equilibrium ground surface temperature $T_{0}$, the constant thermal conductivity $\lambda$, and heat production rate $A$ of the subsurface, which in most cases can safely be neglected. If the GST history is parameterized by a series of temperature steps $T_{j}^{G}$ at times $t_{j}$ before present $(t=0)$, the remaining transient temperature term $T_{t}(z, t)$ at time $t$ and depth $z$ is given by Carslaw and Jaeger (1959):

$$
T_{t}(z)=\sum_{j=1}^{N} T_{j}^{G}\left(\operatorname{erfc}\left(\frac{z}{2 \sqrt{\kappa t_{j}}}\right)-\operatorname{erfc}\left(\frac{z}{2 \sqrt{\kappa t_{j-1}}}\right)\right) .
$$

If $T_{t}(z)=T_{t}\left(z_{i}\right)$ is given at discrete depths $i$, a corresponding linear inverse problem for the $T_{j}^{G}, T_{0}$, and $q_{0}$ can be formulated. In the cases shown, 20 temperature steps logarithmically equispaced between $10 \mathrm{yr} \mathrm{BP}$ and $1000 \mathrm{yr} \mathrm{BP}$ were used. To deal with the inherent ill-posedness of this problem
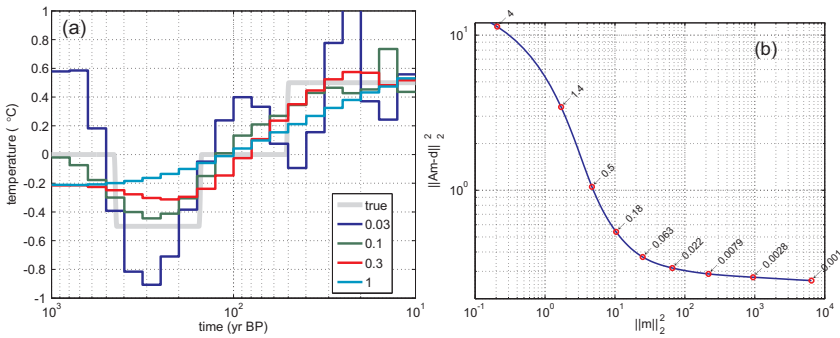

Fig. B2. Inversions of borehole temperature profiles for a fixed depth $(500 \mathrm{~m})$, where a GSTH of constant value before the LIA is assumed. Results for different values of the regularization parameter are shown in (a). The corresponding L-curve (Hansen, 2010) is shown in panel (b).

(Hansen, 2010), it is solved using a truncated singular value decomposition approach as described by Mareschal and Beltrami (1992). In order to keep the numerical experiment free of the ambiguities when choosing the necessary regularization parameter, a constant $\epsilon$ was determined beforehand by the L-curve method (Hansen, 1998, 2010). For all depths considered here, a value of $\epsilon=0.25$ seemed appropriate. Individual determination of the regularization parameter for each temperature profile does not produce fundamentally different results.

To shed light on the generally smoothing behavior of this algorithm, a simple numerical experiment assuming a GSTH of constant value before the LIA is presented here. For the numerical experiments we used the GSTHs shown in Fig. B1, random perturbation were added to the original simulated data, assuming a normal distribution $\mathcal{N}(\hat{\mu}, \hat{\sigma})$ with $\hat{\mu}=0$ and standard deviations $\hat{\sigma}=0.1 \mathrm{~K}$. Noise correlation was produced using a rectangular linear triangular filter of length 5 .

The results for different choices of the regularization parameter $\epsilon$ are given in Fig. B2a. The overall smoothing behavior of this regularized inversion is evident. Additionally, the L-curve for this experiment is shown in Fig. B2b. While at small regularization parameters (e.g. $\epsilon=0.03$, blue), the inverted GST shows overshoots, and a behavior strongly dependent on noise in the observations, higher values (e.g. $\epsilon=0.3$ ) will lead to stable, but oversmoothing behavior. This is well reflected in the L-curve (Hansen, 2010) on the right, which has its corner somewhere between $\epsilon=0.4$ and $\epsilon=0.1$. As common in ill-posed inverse problems, a trade-off between data fit and stability of results can be obtained near the corner of the L-curve.

\section{Supplementary material related to this article is available online at: http://www.clim-past.net/8/1059/ 2012/cp-8-1059-2012-supplement.zip.}


Acknowledgements. We thank J.-C. Mareschal and the anonymous reviewer for their efforts, which improved this article considerably. VR was funded by the Ramón y Cajal Program (Gobierno de España, Ministerio de Educación, Cultura y Deporte), project CCG10-UCM/ESP-5070 (Comunidad de Madrid/UCM), and project CGL2011-29672-C02-01 (Gobierno de España, Ministerio de Economía y Competitividad).

Edited by: B. Vinther

\section{References}

Beltrami, H. and Mareschal, J.-C.: Recent warming in eastern Canada inferred from Geothermal Measurements, Geophys. Res. Lett., 18, 605-608, 1991.

Beltrami, H., Chapman, D. S., Archambault, S., and Bergeron, Y.: Reconstruction of high resolution ground temperature histories combining dendrochronological and geothermal data, Earth Planet. Sc. Lett., 136, 437-445, 1995.

Beltrami, H., Smerdon, J. E., Matharoo, G. S., and Nickerson, N.: Impact of maximum borehole depths on inverted temperature histories in borehole paleoclimatology, Clim. Past, 7, 745-756, doi:10.5194/cp-7-745-2011, 2011.

Birch, F.: The Effects of Pleistocene Climatic Variations upon Geothermal Gradients, Am. J. Sci., 246, 729-60, 1948.

Carslaw, H. S. and Jaeger, J. C.: Conduction of heat in solids, Oxford Univ. Press. 2nd Edn., Oxford,UK, 1959.

Chouinard, C. and Mareschal, J.-C.: Ground surface temperature history in southern Canada: Temperatures at the base of the Laurentide ice sheet and during the Holocene, Earth Planet. Sc. Lett., 277, 280-289, 2009.

Clauser, C. and Mareschal, J.-C.: Ground temperature history in central Europe from borehole temperature data, Geophys. J. Int., 121, 805-817, 1995.

Davies, J. H. and Davies, D. R.: Earth's surface heat flux, Solid Earth, 1, 5-24, doi:10.5194/se-1-5-2010, 2010.

González-Rouco, J. F., Beltrami, H., Zorita, E., and Stevens, M. B.: Borehole climatology: a discussion based on contributions from climate modeling, Clim. Past, 5, 97-127, doi:10.5194/cp-5-972009, 2009.

Hansen, P. C.: Rank Deficient and Discrete Ill-Posed Problems, SIAM, Philadelphia, 1998.

Hansen, P. C.: Discrete Inverse Problems. Insight and Algorithms, SIAM, Philadelphia PA, 2010.

Hartmann, A. and Rath, V.: Uncertainties and shortcomings of ground surface temperature histories derived from inversion of temperature logs, J. Geophys. Eng., 2, 299-311, doi:10.1088/1742-2132/2/4/S02, 2005.

Jansen, E., Overpeck, J., Briffa, R., Duplessy, J.-C., Joos, F., Masson-Delmotte, V., Olago, D., Otto-Bliesner, B., Peltier, W., Rahmstorf, S., Ramesh, R., Raynaud, D., Rind, D., Solomina, O., Villalba, R., and Zhang, D.: Palaeoclimate, in: Climate Change 2007: The Physical Science Basis, edited by: Solomon, S., Qin, D., Manning, M., Chen, Z., Marquis, M., Averyt, K., Tignor, M., and Miller, H., Cambridge University Press, Cambridge, UK and New York, NY, USA, 2007.

Jost, A., Lunt, D., Kageyama, M., Abe-Ouchi, A., Peyron, O., Valdes, P. J., and Ramstein, G.: High-resolution simulations of the last glacial maximum climate over Europe: a solution to discrepancies with continental palaeoclimatic reconstructions, Clim. Dynam., 24, 577-590, doi:10.1007/s00382-005-0009-4, 2005.

Jouzel, J., Masson-Delmotte, V., Cattani, O., Dreyfus, G., Falourd, S., Hoffmann, G., Minster, B., Nouet, J., Barnola, J., Chappellaz, J., Fischer, H., Gallet, J., Johnsen, S., Leuenberger, M., Loulergue, L., Luethi, D., Oerter, H., Parrenin, F., Raisbeck, G., Raynaud, D., Schilt, A., Schwander, J., Selmo, E., Souchez, R., Spahni, R., Stauffer, B., Steffensen, J., Stenni, B., Stocker, T., Tison, J., Werner, M., and Wolff, E.: Orbital and Millennial Antarctic Climate Variability over the Past 800,000 Years, Science, 317, 793-797, 2007.

MacDougall, A. H., Beltrami, H., González-Rouco, J. F., Stevens, M. B., and Bourlon, E.: Comparison of observed and general circulation model derived continental subsurface heat flux in the Northern Hemisphere, J. Geophys. Res, 115, D12109, doi:10.1029/2009JD013170, 2010.

Majorowicz, J. A.: Thermal lithosphere across the Trans-European Suture Zone in Poland, Geol. Q., 49, 1-14, 2004.

Majorowicz, J. and Wybraniec, S.: New terrestrial heat flow map of Europe after regional paleoclimatic correction application, Int. J. Earth Sci., online first: doi:10.1007/s00531-010-0526-1, 2010.

Majorowicz, J., Šafanda, J. S., and Torun-1 Working Group: Heat flow variation with depth in Poland: evidencefrom equilibrium temperature logs in 2.9-km-deep well Torun-1, Int. J. Earth Sci., 97, 307-315, doi:10.1007/s00531-007-0210-2, 2008.

Mareschal, J.-C. and Beltrami, H.: Evidence for recent warming from perturbed thermal gradients: examples from eastern Canada, Clim. Dynam., 6, 135-143, 1992.

Mottaghy, D. and Rath, V.: Latent heat effects in subsurface heat transport modeling and their impact on paleotemperature reconstructions, Geophys. J. Int., 164, 236-245, doi:10.1111/j.1365246X.2005.02843.x, 2006.

NGRIP Working Group: High-resolution record of Northern Hemisphere climate extending into the last interglacial period, Nature, 431, 147-151, 2004.

Otto-Bliesner, B., Schneider, R., Brady, E. C., Kucera, M., AbeOuchi, A., Bard, E., Braconnot, P., Crucifix, M., Hewitt, C. D., Kageyama, M., Marti, O., Paul, A., Rosell-Melé, A., Waelbroeck, C., Weber, S. L., Weinelt, M., and Yu, Y.: A comparison of PMIP2 model simulations and the MARGO proxy reconstruction for tropical sea surface temperatures at last glacial maximum, Clim. Dynam., 32, 799-815, 2009.

Pollack, H. N. and Huang, S.: Climate reconstruction from subsurface temperatures, Annu. Rev. Earth. Pl. Sc., 28, 339-365, 2000.

Rath, V. and Mottaghy, D.: Smooth inversion for ground surface temperature histories: estimating the optimum regularization parameter by generalized cross-validation, Geophys. J. Int., 171, 1440-1448, doi:10.1111/j.1365-246X.2007.03587.x, 2007.

Smerdon, J. E. and Stieglitz, M.: Simulating heat transport of harmonic temperature signals in the Earth's shallow subsurface: Lower-boundary sensitivities, Geophys. Res. Lett., 33, L14402, doi:10.1029/2006GL026816, 2006.

Smerdon, J. E., Pollack, H. N., Cermak, V., Enz, J. W., Kresl, M., Šafanda, J., and Wehmiller, J. F.: Daily, seasonal and annual relationships between air and subsurface temperatures, J. Geophys. Res., 111, D07101, doi:10.1029/2004JD005578, 2006.

Stevens, M. B., González-Rouco, J. F., and Beltrami, H.: North American climate of the last millennium: Underground temper- 
atures and model comparison, J. Geophys. Res., 113, F01008, doi:10.1029/2006JF000705, 2008.

Stieglitz, M. and Smerdon, J.: Characterizing Land-Atmosphere coupling and the implications for subsurface thermodynamics, J. Climate, 20, 21-37, doi:10.1175/JCLI3982.1, 2007.

Tarantola, A.: Inverse problem theory. Methods for model parameter estimation, SIAM, Philadelpha, available at: http://www. ipgp.jussieu.fr/ $\sim$ tarantola/Files/Professional/Books/index.html (last access: 18 June 2012), 2005.

Vasseur, G. and Lucazeau, F.: Bounds on paleotemperatures and paleoclimatic corrections, ZBL Geo. Pal., 1, 17-24, 1983.
Verdoya, M., Chiozzi, P., and Pasquale, V.: Thermal log analysis for recognition of ground surface temperature change and water movements, Clim. Past, 3, 315-324, doi:10.5194/cp-3-315-2007, 2007.

Wilhelm, H., Popov, Y., Burkhardt, H., Šafanda, J., Čermák, V., Heidinger, P., Korobkov, D., Romushkevich, R., and Mayr, S.: Heterogeneity effects in thermal borehole measurements in the Chicxulub impact crater, J. Geophys. Eng., 2, 357-363, doi:10.1088/1742-2132/2/4/S09, 2005. 